\title{
Three Dimensional Temporal and Spatial Distribution of Adults of Tribolium castaneum (Coleoptera: Tenebrionidae) in Stored Wheat Under Different Temperatures and Adult Densities
}

\author{
Fuji Jian • Ron Larson • Digvir S. Jayas • \\ Noel D. G. White
}

Received: 23 November 2011 / Accepted: 20 January 2012/Published online: 8 March 2012

(C) NAAS (National Academy of Agricultural Sciences) 2012

\begin{abstract}
Three dimensional temporal and spatial distributions of adults of Tribolium castaneum (Herbst) at insect densities of 0.1 (low), 1.0 (medium), and 5.0 (high) adults $/ \mathrm{kg}$ wheat $(\mathrm{A} / \mathrm{kg}$ ) were determined in a $1.5 \mathrm{t}$ bin of wheat with $11 \pm 0.8 \%, 13 \pm 0.6 \%$ and $15 \pm 0.3 \%$ moisture contents (wet basis) at $20 \pm 1,25 \pm 1$ and $30 \pm 1{ }^{\circ} \mathrm{C}$. The $1.5 \mathrm{t}$ of wheat was sampled at five locations. At each location, grain was separated into three $15 \mathrm{~kg}$ vertical layers and adult numbers in each layer were counted. Adults did not prefer any location in the horizontal direction, while fewer adults were recovered from the bottom layers than that from the top and middle layers. Number of adults at a location with any temperature or insect density did not correlate with its adjacent locations in either vertical or horizontal direction. The temporal continuous property existed at all insect densities. These results were consistent with the tendency of the aggregation of the adults at the low and medium densities. Adults had highly clump distributions at low and medium densities and this aggregation behavior decreased with the increase of insect densities. Temperature tested did not influence their aggregation behavior.
\end{abstract}

Keywords Population density $\cdot$ Spatial distribution $\cdot$ Temporal continuity $\cdot$ Tribolium castaneum

\section{Introduction}

Three-dimensional temporal and spatial distributions are one of the most important ecological properties of a species. The characterized patterns of three-dimensional temporal and spatial distributions of insects can be used to effectively manage insect pests in stored grain such as sampling and insect control. The red flour beetle, Tribolium castaneum (Herbst) (Coleoptera: Tenebrionidae), is an economically

F. Jian · D. S. Jayas $(\bowtie)$

Department of Biosystems Engineering, University of Manitoba, Winnipeg, MB R3T 5V6, Canada

e-mail: Digvir_jayas@umanitoba.ca

F. Jian · R. Larson

OPIsystems Inc., 1216 36th Avenue NE, Calgary, AB T2E 6M8, Canada

N. D. G. White

Agriculture and Agri-Food Canada, Cereal Research Centre, 195 Dafoe Road, Winnipeg, MB R3T 2M9, Canada important insect pest of stored products in the world [26, 33]. Adults of T. castaneum move inside stored grain bulks and respond to temperature gradients $[3,13,16,19,36-39]$ and different habitats $[23,28,43]$. There is no report on the temporal distribution of $T$. castaneum in grain bulks. There have been some efforts to describe their spatial distribution in stored grain $[6,15,28,36-39,43]$ and aggregation in the top part of grain bulks has been reported [39]. However, all of the reports are based on either small sample units of grain $(<2.75 \mathrm{~kg})$, small containers $(<25 \mathrm{~kg}$ grain), or without grain. Small sample units can increase edge effect [9] and might not be able to detect insects [20]. There is no study on spatial distribution of $T$. castaneum using large sample units under large scale laboratory conditions. The insect density inside the grain in these reports is also not known. With these small sample units and unknown insect densities or inside small containers, it is not known if the characterized spatial distribution of adults of $T$. castaneum is accurate. To understand their spatial distribution parameters, large scale laboratory tests with large sample units and known insect densities are required. 
Advances in the analysis of spatial data by using geostatistical tools to quantify and model spatial and temporal correlations, have allowed for quantitative analyses of insect spatial and temporal patterns on both small and large scales [29]. Geostatistical methods are used to characterize spatial variability of insect emergence patterns [10], presence and density [22], and spatial concurrence of adults with known breeding habitats [25]. Geostatistical techniques of correlogram and Kriging, based on semivariogram properties, can provide interpolated values for response variables, such as insect population density, at locations not actually sampled. Applied to insect pest populations, these techniques may allow prediction of areas in fields where pest populations may be expected to exceed an action threshold [10, 22, 30]. Jian et al. [20] characterized the temporal and spatial distribution of adults of Cryptolestes ferrugineus inside stored wheat. The concurrence continuity of adults of $T$. castaneum in three dimensions at both spatial and time scale is not known.

The objective of this study was to characterize the spatial and temporal distribution patterns of the adults of T. castaneum in three dimensions under different introduced insect densities, grain moistures, and temperatures by using large sampling units ( $15 \mathrm{~kg}$ of grain per unit and about $15 \%$ of total grain bulk in the test bin was sampled).

\section{Materials and Methods}

\section{Test Bin and Grain}

Plastic bins ( $1.5 \mathrm{~m}$ diameter and $1.5 \mathrm{~m}$ high) filled with about $1.5 \mathrm{t}$ wheat were used (Fig. 1). To prevent insects from escaping, bins were covered loosely with lids and one layer of liquid Teflon was applied on the inside wall and below the top rim of the bins (about $10 \mathrm{~cm}$ high) [20]. Hard red spring wheat (AC Barrie) was purchased from a grain elevator with $11.0 \pm 0.8 \% \quad(n=12)$, or $13.0 \pm 0.6 \%$ $(n=24)$ moisture contents and $<0.6 \pm 0.3 \%$ dockage $(n=36)$. The dockage included $0.4 \pm 0.1 \%$ small materials (smaller than $2 \mathrm{~mm}$ diameter consisting of small stones, weed seeds, and small foreign material) and $0.2 \pm 0.1 \%$ large material (larger than $4.75 \mathrm{~mm}$, consisting of wheat chaff and straw). During the pre-experiment, dead adults of $C$. ferrugineus and $T$. castaneum were found inside the delivered wheat. The density of the dead insects was $<0.001 \mathrm{~A} / \mathrm{kg}$ wheat.

\section{Insects}

The cultures of $T$. castaneum, obtained from the Cereal Research Center, Agriculture and Agri-Food Canada, Winnipeg, were reared in the laboratory at room

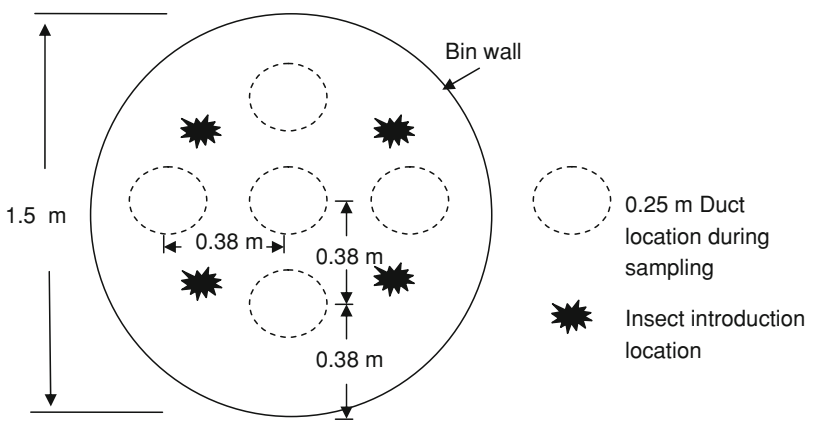

Fig. 1 Experimental set up (top view)

temperature and $70 \pm 5 \% \mathrm{RH}$ for $2-3$ months. The feed was the same whole wheat as used in the testing. Adults were selected using a gentle vacuum. The selected adults were kept inside four 4-L glass bottles with about three kilograms of the same wheat in each bottle. Before introduction of the insects, the bottles were kept inside the test bin for at least $24 \mathrm{~h}$ allowing the insects to acclimate to the experimental conditions.

\section{Experimental Treatments}

Materials and methods are similar to the detail reported in Jian et al. [20] and reported here for easy reference and clarity. Experiments were conducted at 20,25 , and $30^{\circ} \mathrm{C}$; and in stored wheat at $11 \%, 13 \%$ and $15 \%$ moisture contents (wet basis) [5]. The method of grain temperature and moisture content control is the same as reported by Jian et al. [20]. There were a total of nine experiments and no replicates were done due to the scale of the study and the difficulty of bringing the initial grain temperature (at room temperature) up to the desired temperature $\left(25\right.$ or $\left.30^{\circ} \mathrm{C}\right)$ without losing grain moisture. At each grain temperature and moisture content, three insect densities (0.1 (low), 1.0 (medium), and 5.0 (high) adults $/ \mathrm{kg}$ of wheat, referred to as $\mathrm{A} / \mathrm{kg}$ ) were introduced. During the data analysis, it was found moisture did not influence the pattern of the spatial and temporal distribution of the adults. Therefore, data at different grain moisture contents but at the same temperature or introduced insect density were pooled and treated as replicates at the same temperature. After this pooling, there were three replicates at each temperature or insect density.

\section{Test Procedure}

Grain was loaded to $1.1 \mathrm{~m}$ height in the test bins and the grain surface was leveled. After the grain temperature was stable and uniformly distributed, 150 adults of T. castaneum were introduced at the surface of the test grain at four locations (Fig. 1), making an overall insect density of $0.1 \mathrm{~A} / \mathrm{kg}$ (low density). One week after the first 
introduction of the insects, grain was sampled at five locations (three layers at each location, Fig. 1). After counting of the adults inside the grain samples, the grain and the recovered insects were returned to their original locations. One hour after the return of the grain and insects, an additional 1,350 adults (1.0 A/kg, medium density) were introduced. After another 7 days, the previous procedure (including sampling and insect counting) was repeated and an additional 6,000 adults $(5.0 \mathrm{~A} / \mathrm{kg}$, high density) were introduced. After a further 7 days, the grain was removed and the adults were counted again. The experiment was terminated after the third count of the insects. Prior to a test, selected insects were divided equally into four 4-L bottles and introduced at four locations during each introduction time (Fig. 1). During the experimental period the insect mortality was $<1 \%$ and only the number of live adults was used in the data analysis.

\section{Sampling and Insect Recovery}

The method reported by Jian et al. [20] was used. To test the shaker efficiency, the following pre-experiment was conducted. One hundred adults were introduced into a bucket with $15 \mathrm{~kg}$ wheat (the same wheat with $13 \% \mathrm{MC}$ ). After the insect introduction, the bucket was sealed by using duct tape and kept at room temperature. One week or $3 \mathrm{~h}$ later, adults were separated from the wheat by using the shaker. The recovery rate of the adults was $\geq 98 \%(n=6)$ for the $3 \mathrm{~h}$ treatment and $\geq 97 \%(n=6)$ for 1 week treatment. During data analysis, the count number of recovered adults was converted by using the following equation:

Insect number in the sample

$=$ Counted insect number in the sample $\times 1.03$

Data Analysis

A total of 27 sampling sets were collected in the entire study. There were 15 samples (each about $15 \mathrm{~kg}$ of wheat) in each sampling set and each of these 15 samples was referred to as one unit. The data of adult densities at each temperature or introduced insect density were used to do the geostatistical analysis. Correlation coefficient and covariance [40] between the following pairs of recovered insect densities were calculated: $H(0,38) ; H(0,54) ; V(0,37) ; V(\mathrm{~T}, \mathrm{M}) ; V(\mathrm{M}$, B); and $V(0,74)$; where $H$ and $V$ were the horizontal and vertical direction, respectively. The second number inside each bracket was the distance between two sample locations and the first number was the starting location. The first number " 0 " indicated the starting location at the center of the bins. T, M, and B were the top, middle, and bottom layer, respectively. A variogram was prepared at each introduced insect density [12]. To test if the previous insect densities would influence the distribution of the following introduced insects, Spearman's coefficient of rank correlation was determined [8], the correlation coefficient was calculated and its significance was tested [31].

A frequency table was prepared by ranking the collected insect numbers in each sample unit into ten groups. This category was classified using a class width of $2.5,16.0$, and 30.0 counts of the adults when introduced density was 0.1 , 1.0 , and $5.0 \mathrm{~A} / \mathrm{kg}$, respectively. The class width was calculated by dividing the range between the highest and lowest insect counts at each introduced insect density into ten equal parts. Frequency distribution for sampling sets at the same temperature or introduced insect density was examined for the one-way frequency table. The Chi-square test and Lagrange multiplier statistics for type three analyses, were applied at $\alpha=0.05$ level. We treated the ten groups as ordinal data $[1,2]$ and Proc GENMOD in SAS 9.2 [31] was used to conduct the normal and Poisson distribution tests. The negative binomial and binomial distribution were tested using the numbers of sample units with zero adult count and non zero count in sample units at the same temperature or same introduced insect density.

To test the degree of aggregation of the adults, Morisita index, $b_{0}$ and $b_{1}$ values associated with Lloyd's index of mean crowding [17, 18, 24], and $\ln a$ and $b$ values associated with the Taylor's power law were calculated [14, 20]. The significance of the calculated Morisita's index was assessed by comparing the calculated Chi-square with the critical value of the Chi-square distribution [34]. The value of $b_{0}$ was used to estimate the size of clumps of individuals. The value of $b_{1}>1$ indicates a series of distributions with constant tendency for aggregation. The value of $b$ was used to categorize uniform, random, and aggregated spatial patterns of insect distribution based on $b$ values that were $<1,1$, and $>1$, respectively $[21,35,44]$. There was no significant difference in the $b$ values at different insect densities, temperatures, and moisture contents. Therefore, all the data were pooled and the overall $\ln a$ and $b$ were calculated.

\section{Results and Discussion}

\section{Three-Dimensional Distribution}

The introduced adults were recovered from all layers after 7 days (Figs. 2, 3). In the horizontal direction, adults did not prefer any location (Fig. 4) because 122, 53, and 149 out of 324 sample units had higher, equal to, or lower insect density at the center locations than that at half radius locations. The distribution pattern in the horizontal direction of the adults at low density was not different from that at medium and high densities (Fig. 4). In the vertical 

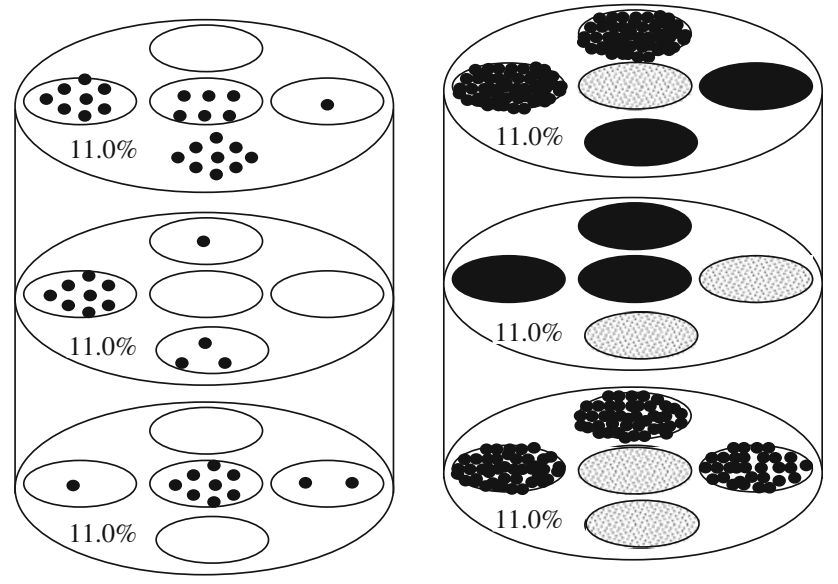

Fig. 2 Adult $T$. castaneum redistribution at $20 \pm 1{ }^{\circ} \mathrm{C}$ and in $11 \pm 0.8 \% \mathrm{MC}$ wheat without temperature and moisture gradients. The introduced insect density was 0.1 (left) and 5.0 (right) $\mathrm{A} / \mathrm{kg}$. In the figure, numbers show the wheat moisture contents at each layer. One dot represents one adult. Dark and hatched circles show the number of adults at the sample location was $>90$ or between 60 and 90 , respectively

direction, the adults preferred the top two layers at all insect densities (Fig. 5) because 12 out of 108 sample units at the bottom layer had lower insect density than that at the middle layer. Adult densities at the middle layer had $60 \%$, $11 \%$, and $28 \%$ higher, equal to, or lower than that at the top layer (Fig. 5). This slight high adult density at the middle layer might not be caused by the small moisture difference because there was no moisture gradient at $20^{\circ} \mathrm{C}$. These results indicated that the adults prefer to stay in the top $0.7 \mathrm{~m}$ of the grain bulk.

Surtees [36] found the number of the adults, introduced at the top of the grain container, decreased from top to bottom. Jian et al. [19] found more than $90 \%$ of the adults, introduced at the middle of a 1 meter wheat column, were distributed in the middle of the column. They proposed that the small inter-granular spaces in a wheat bulk relative to the size of the insect limit the movement ability of the beetles in both horizontal and vertical directions. The adult distribution pattern in this study supported this suggestion.

\section{Spatial and Temporal Continuity}

Covariance increased with the increase of the insect densities, while it did not increase or decrease with the increase of temperature and distance in both the vertical and horizontal directions (Tables 1,2). Correlation coefficient did not change with the change of temperature, density and distance (Tables 1,2). These results indicated that numbers of insects at any temperature, location or density were not correlated with its adjacent locations in both vertical and horizontal directions (Figs. 2, 3).

Spearman's coefficient of rank correlation showed that 5 out of 6 cases (83\%) indicated autocorrelations between the current and its previous insect density at the same locations (Table 3). The only one with no significant autocorrelation was at $25^{\circ} \mathrm{C}$ and between the low and medium insect densities, while there was a significant autocorrelation at this temperature and between the medium and high introduced insect densities. Therefore, the temporal continuous property might exist. The temporal continuity of the adults of $T$. castaneum might relate to the aggregation pheromone released by males to attract females [7, 11]. Adults of T. castaneum also produce different volatile compounds and the amount of these compounds produced varies with insect density and temperature [4, 32]. These volatiles might also cause adults to aggregate [4]. This conclusion indicated that density trials in this study were dependent when the beetles were added to the grain sequentially to bring the grain to higher insect densities, and adults might aggregate in stored grain bins.
Fig. 3 Adult $T$. castaneum redistribution at $30 \pm 1{ }^{\circ} \mathrm{C}$ and in $15 \pm 0.1 \% \mathrm{MC}$ wheat without temperature gradient and with moisture gradients. The introduced insect density was 0.1 (left), 1.0 (middle), and 5.0 (right) $\mathrm{A} / \mathrm{kg}$. In the figure, numbers show the wheat moisture contents at each layer. One dot represents one adult. Dark and hatched circles show the number of adults at the sample location was $>90$ or between 60 and 90, respectively
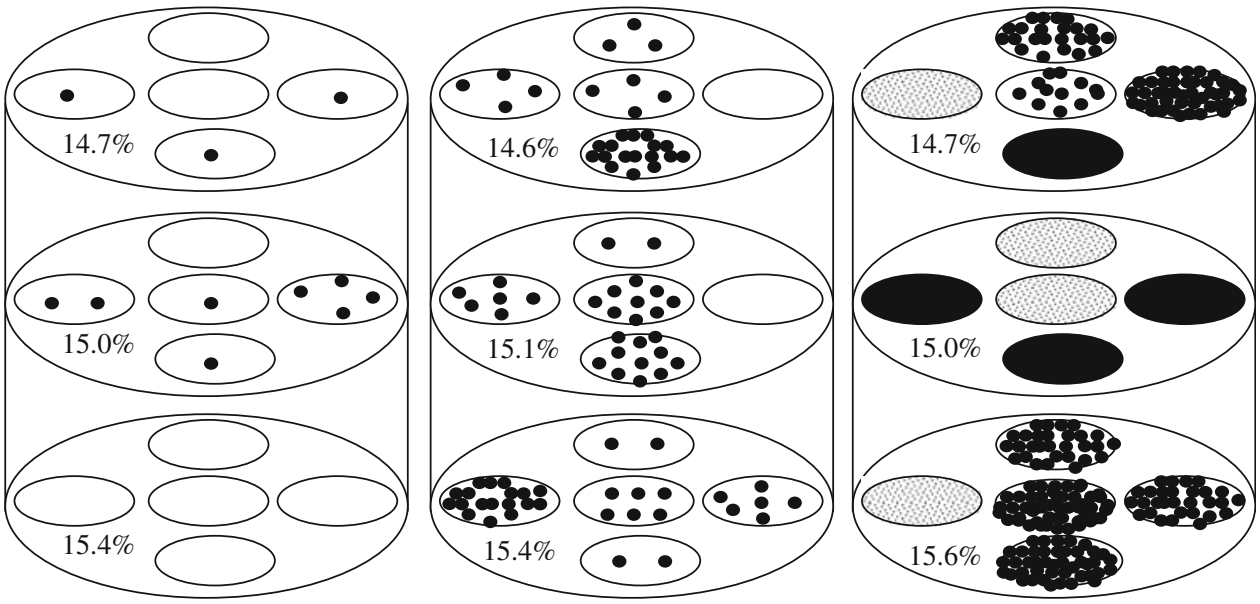
Fig. 4 h-Scatterplots for 38 (left) and $54 \mathrm{~cm}$ (right) separation distance in the horizontal direction for adult T. castaneum. Line is the $45^{\circ}$ bisector. The introduced insect density was 0.1 (top), 1.0 (middle), and 5.0 (bottom) $\mathrm{A} / \mathrm{kg}$
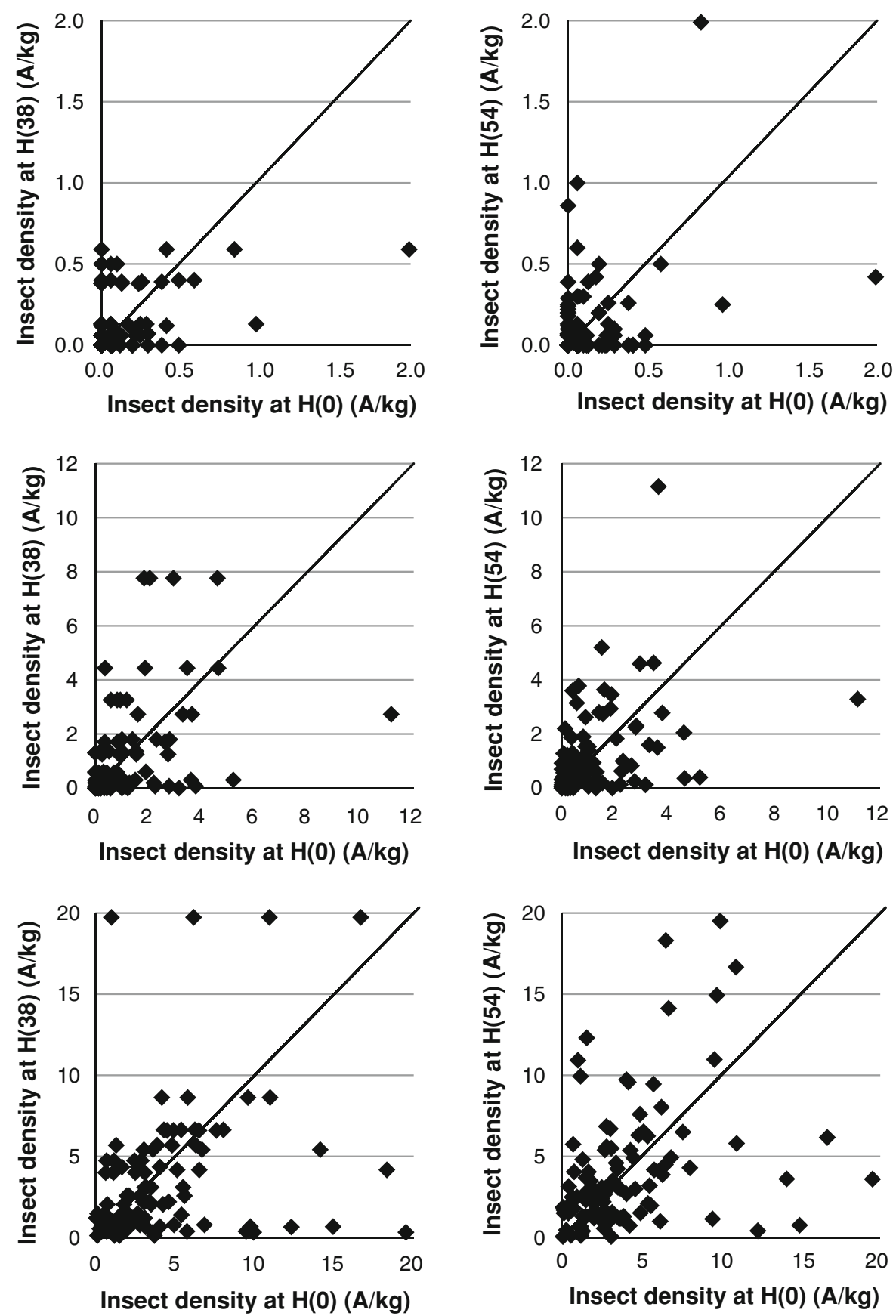

Obtaining reliable models of spatial autocorrelation typically requires many samples (e.g., a minimum 50-100 observations) [20, 27, 42]. Collecting samples with a large distance between samples and without the knowledge of the continuity of insects in three dimensions at both spatial and time scales might result in a wrong explanation of spatial distribution of the insects $[8,20]$. The best approach is to sample all the grain inside a test bin but this may not be practical because sampling and counting insects in a short time period (say $<1$ day) might be a challenge [20]. In this study, we sampled about $15 \%$ of the wheat in the
$1.5 \mathrm{~m}$ diameter and $1.5 \mathrm{~m}$ high bin. Even though there were only 15 units in each sampling set, there were a total of 405 sampling units and the mass of each unit was $15 \mathrm{~kg}$. Therefore, to characterize the concurrence continuity of adults of $T$. castaneum at different temperatures or insect densities at both spatial and time scales, using these collected data should be acceptable.

The fact of the temporal continuity might be used to develop a sample or detection plan of the adults of T. castaneum using their aggregation behavior (such as in a pheromone trap) in grain bins. However, the fact of 
Fig. 5 h-Scatterplots for $37 \mathrm{~cm}$ separation distance in the vertical direction. The left graphs show adult $T$. castaneum density between the locations at top and middle layers, and the right graphs show the density between the locations at middle and bottom layers. Line is the $45^{\circ}$ bisector. The introduced insect density was 0.1 (top), 1.0 (middle), and 5.0 (bottom) $\mathrm{A} / \mathrm{kg}$
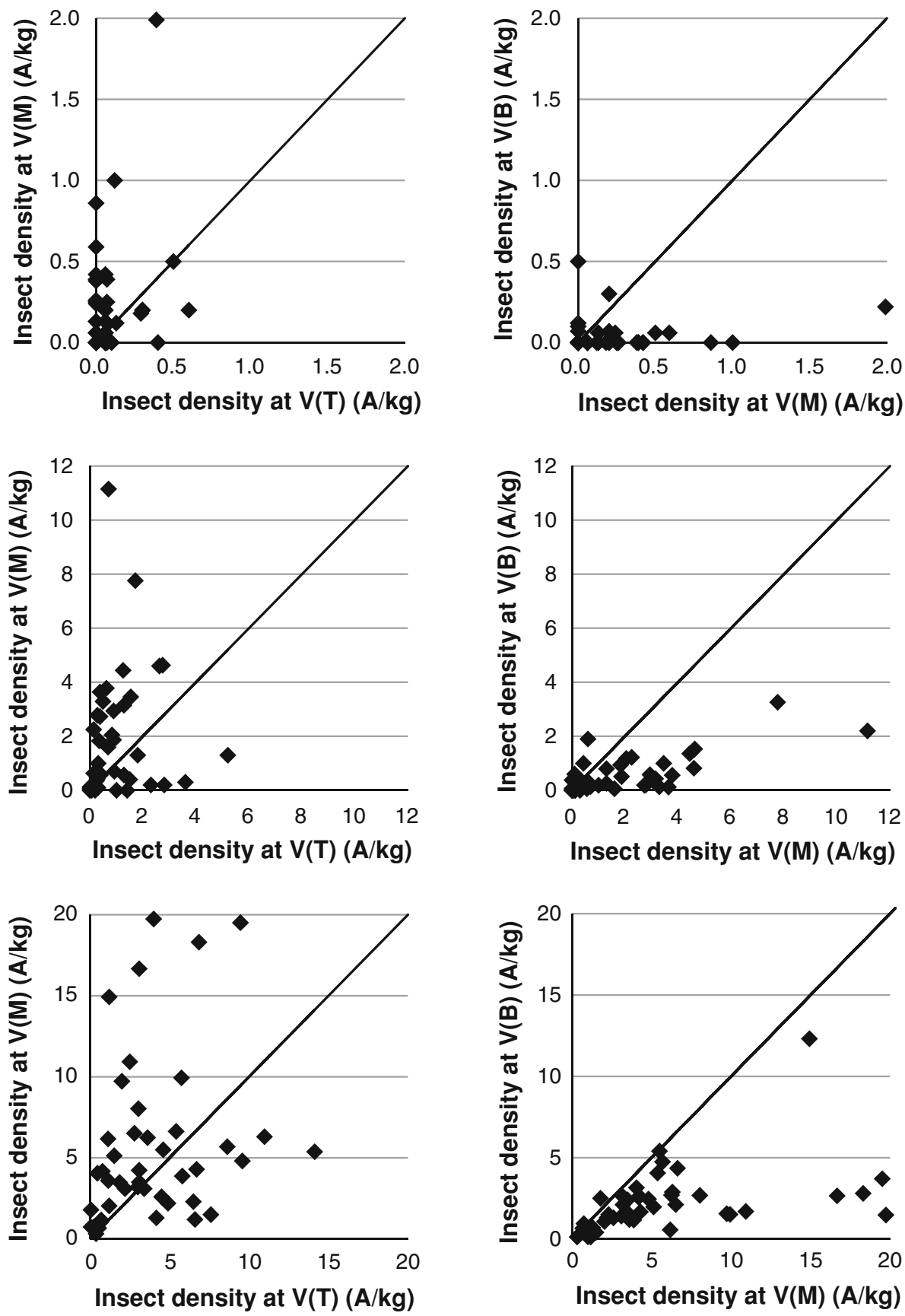

non-autocorrelation at any insect density, temperature, and distance might suggest that using sampling techniques to estimate densities of $T$. castaneum at the adjacent locations of the sampled places might not work.

\section{Distribution of Count Frequency}

The normal distribution and Poisson models were not appropriate for describing the count frequency in all of the sampling sets (Table 4). This indicated the adults were not randomly distributed. The count frequency fitted the model of negative binomial and binomial in 78 and $44 \%$ of the time, respectively (Table 4 ). This summarized the fact that there was a high probability of zero adults in sample units at the low insect density and there was low probability at the high insect density. When the insect counts at the same introduced insect density were pooled, the frequency distribution pattern was the same as the above characterization summarized, i.e., adults were not randomly distributed and there was a high probability of zero adults in sample units at the low insect density. 
Table 1 Correlation coefficient and covariance of the densities of adult $T$. castaneum at different locations and in the horizontal direction in wheat bulks

\begin{tabular}{|c|c|c|c|c|c|c|c|}
\hline \multirow{3}{*}{$\begin{array}{l}\text { Insect } \\
\text { density } \\
(\mathrm{A} / \mathrm{kg})\end{array}$} & \multirow{3}{*}{$\begin{array}{l}\text { Geostatistical } \\
\text { pair }\end{array}$} & \multicolumn{6}{|c|}{ Grain temperature $\left({ }^{\circ} \mathrm{C}\right)$} \\
\hline & & \multicolumn{2}{|l|}{20} & \multicolumn{2}{|l|}{25} & \multicolumn{2}{|l|}{30} \\
\hline & & $R^{\mathrm{a}}$ & $V^{\mathrm{b}}$ & $R^{\mathrm{a}}$ & $V^{\mathrm{b}}$ & $R^{\mathrm{a}}$ & $V^{\mathrm{b}}$ \\
\hline \multirow[t]{3}{*}{0.1} & $H(0,38)$ & 0.305 & $0.006 \pm 0.008$ & 0.632 & $0.032 \pm 0.030$ & 0.124 & $0.002 \pm 0.001$ \\
\hline & $H(0,54)$ & 0.320 & $0.010 \pm 0.007$ & 0.372 & $0.042 \pm 0.041$ & -0.009 & $0.000 \pm 0.001$ \\
\hline & $H(0,76)$ & -0.054 & $0.000 \pm 0.006$ & 0.062 & $0.015 \pm 0.017$ & 0.489 & $0.004 \pm 0.002$ \\
\hline \multirow[t]{3}{*}{1.0} & $H(0,38)$ & 0.358 & $1.817 \pm 0.400$ & 0.169 & $0.130 \pm 0.135$ & 0.108 & $-0.005 \pm 0.004$ \\
\hline & $H(0,54)$ & 0.365 & $1.281 \pm 0.811$ & 0.473 & $0.526 \pm 0.207$ & 0.144 & $0.211 \pm 0.108$ \\
\hline & $H(0,76)$ & 0.187 & $0.965 \pm 0.544$ & 0.473 & $0.452 \pm 0.223$ & 0.731 & $0.642 \pm 0.687$ \\
\hline \multirow[t]{3}{*}{5.0} & $H(0,38)$ & 0.535 & $10.784 \pm 8.266$ & 0.128 & $1.916 \pm 1.745$ & 0.590 & $1.709 \pm 1.507$ \\
\hline & $H(0,54)$ & 0.448 & $6.502 \pm 2.652$ & 0.234 & $5.509 \pm 3.798$ & 0.578 & $9.179 \pm 5.735$ \\
\hline & $H(0,76)$ & 0.021 & $3.300 \pm 1.145$ & 0.038 & $3.487 \pm 1.331$ & 0.231 & $4.125 \pm 3.380$ \\
\hline
\end{tabular}

${ }^{\text {a }}$ Correlation coefficient

b Covariance (mean $\pm \mathrm{SE}$ )

Table 2 Correlation coefficient and covariance of the densities of adult T. castaneum at different locations and in the vertical direction in wheat bulks

\begin{tabular}{|c|c|c|c|c|c|c|c|}
\hline \multirow{3}{*}{$\begin{array}{l}\text { Insect } \\
\text { density } \\
(\mathrm{A} / \mathrm{kg})\end{array}$} & \multirow{3}{*}{$\begin{array}{l}\text { Geostatistical } \\
\text { pair }\end{array}$} & \multicolumn{6}{|c|}{ Grain temperature $\left({ }^{\circ} \mathrm{C}\right)$} \\
\hline & & \multicolumn{2}{|l|}{20} & \multicolumn{2}{|l|}{25} & \multicolumn{2}{|l|}{30} \\
\hline & & $R^{\mathrm{a}}$ & $V^{\mathrm{b}}$ & $R^{\mathrm{a}}$ & $V^{\mathrm{b}}$ & $R^{\mathrm{a}}$ & $V^{\mathrm{b}}$ \\
\hline \multirow[t]{2}{*}{0.1} & $V(0,37)$ & -0.085 & $-0.004 \pm 0.008$ & 0.036 & $-0.021 \pm 0.015$ & 0.062 & $0.000 \pm 0.003$ \\
\hline & $V(0,74)$ & 0.386 & $0.002 \pm 0.002$ & 0.848 & $0.004 \pm 0.004$ & 0.463 & $0.002 \pm 0.002$ \\
\hline \multirow[t]{2}{*}{1.0} & $V(0,37)$ & -0.131 & $-0.790 \pm 0.806$ & 0.041 & $0.019 \pm 0.197$ & 0.388 & $0.110 \pm 0.067$ \\
\hline & $V(0,74)$ & 0.458 & $0.202 \pm 0.086$ & -0.061 & $0.036 \pm 0.021$ & 0.030 & $-0.167 \pm 0.154$ \\
\hline \multirow[t]{2}{*}{5.0} & $V(0,37)$ & -0.165 & $-3.409 \pm 5.593$ & 0.334 & $7.258 \pm 3.419$ & 0.194 & $-0.825 \pm 1.658$ \\
\hline & $V(0,74)$ & 0.622 & $1.649 \pm 0.462$ & -0.069 & $1.598 \pm 1.436$ & 0.591 & $0.953 \pm 0.283$ \\
\hline
\end{tabular}

${ }^{\text {a }}$ Correlation coefficient

b Covariance (mean $\pm \mathrm{SE})$

Table 3 Statistics of the Spearman's coefficient of rank correlation

Temperature $\left({ }^{\circ} \mathrm{C}\right)$ First-second introduced Second-third insects $^{\mathrm{a}} \quad$ introduced insects ${ }^{\mathrm{a}}$

$\bar{R} \quad P>|R| \quad P>|R|$

\begin{tabular}{lcccc}
\hline 20 & 0.598 & $<0.0001 * * *$ & 0.677 & $<0.0001 * * *$ \\
25 & 0.293 & 0.0505 & 0.649 & $<0.0001 * * *$ \\
30 & 0.308 & $0.0398^{*}$ & 0.533 & $0.0002 * *$ \\
\hline
\end{tabular}

a The test of the Spearman's coefficient of rank correlation was conducted between the first and the second batches or between the second and third batches of introduced insects, respectively

\section{Spatial Distribution}

Morisita's indices were significantly $>1$ (Table 5 ). The $b_{1}$ from the regression of Lloyd were significantly larger than 1 (Student $t$ test, $t=1.580, P=0.153$ ) except at the high density (Table 6). The $b_{1}$ at the low insect density was 5 - to 48-fold larger than that at other two insect densities (Table 6). These results confirmed the tendency of aggregation of the adults and the strong aggregation behavior at low density. The $b_{1}$ at different temperatures was in the range of 1.6-2.4 and smaller than that at the low insect density. The $b_{0}$ was not always $<0$ (Table 6). These results suggested that temperature did not influence their aggregation behavior and a tendency of repelling between individuals might only exist when adult density was high.

The $b$ value from Taylor's regression was $>1$ at different temperatures and introduced insect densities except at high introduced insect density (Table 7). The $b$ value is a speciesspecific attribute and remains constant for the same organism in the same environment [41]. The $b$ value at the low insect density was 6.6-fold higher than at the high insect density (Table 7). This confirmed the conclusions from the calculated Morisita's indices and regression of Lloyd. 
Table 4 Fitted distribution models to frequency data based on counts of adult $T$. castaneum in grain sample units at different temperatures and insect densities

\begin{tabular}{|c|c|c|c|c|c|}
\hline \multirow[t]{2}{*}{ Grain temperature $\left({ }^{\circ} \mathrm{C}\right)$} & \multirow[t]{2}{*}{ Insect density $(\mathrm{A} / \mathrm{kg})$} & \multicolumn{4}{|c|}{ Fitted distribution model ${ }^{\mathrm{a}}$} \\
\hline & & Normal & Negative binomial & Binomial & Poisson \\
\hline \multirow[t]{3}{*}{20} & 0.1 & $5268.58 * * *$ & 0.02 & $62.36 * * *$ & $268.09 * * *$ \\
\hline & 1.0 & $2664.58 * * *$ & 0.00 & 0.00 & $232.26 * * *$ \\
\hline & 5.0 & $565.24 * * *$ & 0.00 & 0.00 & $86.60 * * *$ \\
\hline \multirow[t]{3}{*}{25} & 0.1 & $8291.64 * * *$ & 0.19 & $62.18 * * *$ & $440.97 * * *$ \\
\hline & 1.0 & $7180.58 * * *$ & $10.45^{* *}$ & $42.12 * * *$ & $373.69 * * *$ \\
\hline & 5.0 & $4855.20 * * *$ & 0.00 & 0.00 & $373.47 * * *$ \\
\hline \multirow[t]{3}{*}{30} & 0.1 & $6006.31 * * *$ & 0.02 & $62.36 * * *$ & $274.50 * * *$ \\
\hline & 1.0 & $6751.20 * * *$ & $10.87 * *$ & $38.90 * * *$ & $316.73 * * *$ \\
\hline & 5.0 & $1594.31 * * *$ & 0.00 & 0.00 & $135.34 * * *$ \\
\hline \multirow[t]{3}{*}{ Overall $^{\mathrm{b}}$} & 0.1 & $192169 * * *$ & 0.01 & $187.08 * * *$ & $3295.42 * * *$ \\
\hline & 1.0 & $191012 * * *$ & $34.03 * * *$ & $94.18 * * *$ & $3790.48 * * *$ \\
\hline & 5.0 & $34355.7 * * *$ & 0.00 & 0.00 & $1329.69 * * *$ \\
\hline
\end{tabular}

Table 5 Morisita index $\left(I_{\delta}\right)$ of adult $T$. castaneum under different temperatures and introduced insect densities in wheat bulks

\begin{tabular}{|c|c|c|c|c|c|c|}
\hline \multirow{3}{*}{$\begin{array}{l}\text { Insect } \\
\text { density } \\
\text { (A/kg) }\end{array}$} & \multicolumn{6}{|c|}{ Grain temperature $\left({ }^{\circ} \mathrm{C}\right)$} \\
\hline & \multicolumn{2}{|l|}{20} & \multicolumn{2}{|l|}{25} & \multicolumn{2}{|l|}{30} \\
\hline & $I_{\delta}^{\mathrm{a}}$ & Chi-square $^{\mathrm{b}}$ & $I_{\delta}^{\mathrm{a}}$ & Chi-square $^{b}$ & $I_{\delta}^{\mathrm{a}}$ & Chi-square ${ }^{b}$ \\
\hline 0.1 & 2.5 & 166.71 & 6.3 & 567.21 & 2.0 & 93.78 \\
\hline 1.0 & 2.2 & 1547.17 & 2.9 & 908.53 & 3.4 & 1005.52 \\
\hline 5.0 & 1.7 & 2362.62 & 2.5 & 3505.66 & 2.1 & 2628.01 \\
\hline
\end{tabular}

${ }^{\mathrm{a}} I_{\delta}>1$, aggregated distribution pattern; $I_{\delta}=1$, random; and $I_{\delta}<1$ regular

b All the calculated Chi-square values were greater than the critical value of the Chi-square distribution (18.493) at 44 degree of freedom with Chi-square $=0.05$

Table 6 Parameter estimates $\left(b_{0} \pm \mathrm{SE}, b_{1} \pm \mathrm{SE}\right)$ and coefficient of determination $\left(R^{2}\right)$ from the regression of Lloyd's index of mean crowding on mean density of adults of $T$. castaneum

\begin{tabular}{lcll}
\hline & $b_{0} \pm \mathrm{SE}$ & $b_{1} \pm \mathrm{SE}$ & $R^{2}$ \\
\hline Insect density $(\mathrm{A} / \mathrm{kg})$ & & & \\
0.1 & -1.616 & 9.837 & 0.723 \\
1.0 & 0.00588 & 1.695 & 0.993 \\
5.0 & 6.500 & 0.203 & 0.07 \\
Temperature $\left({ }^{\circ} \mathrm{C}\right)$ & & & \\
20 & -0.473 & 1.654 & 0.985 \\
25 & -0.444 & 2.362 & 0.999 \\
30 & -0.537 & 1.994 & 0.987 \\
\hline
\end{tabular}

There was no moisture gradient in the tests at $20^{\circ} \mathrm{C}$. However, there was a small moisture gradient (about $0.7 \%$ percentage point per meter) at 25 or $30^{\circ} \mathrm{C}$ in the vertical direction, but there was no moisture gradient in the horizontal direction inside the test bins (Figs. 2, 3). Moisture
Table 7 Parameter estimates and coefficient of determination $\left(R^{2}\right)$ from the regression of Taylor's power law

\begin{tabular}{lccc}
\hline & $\ln a \pm \mathrm{SE}$ & $b \pm \mathrm{SE}$ & $R^{2}$ \\
\hline Insect density $(\mathrm{A} / \mathrm{kg})$ & & & \\
0.1 & -0.0508 & 1.369 & 0.887 \\
1.0 & 0.411 & 1.285 & 0.887 \\
5.0 & 2.537 & 0.206 & 0.089 \\
Temperature $\left({ }^{\circ} \mathrm{C}\right)$ & & & \\
20 & 0.204 & 1.734 & 0.998 \\
25 & 0.813 & 1.578 & 0.991 \\
30 & 0.472 & 1.874 & 0.991 \\
\hline
\end{tabular}

contents at each measured location were constant during the experimental period. By comparing the data associated with insect densities at $20^{\circ} \mathrm{C}$ with data at 25 or $30^{\circ} \mathrm{C}$, there was no significant difference in correlation coefficient (Paired $t$ test and all of $P$ values $\geq 0.463$, Table 2 ), the Spearman's coefficient of rank correlation (Paired $t$ test and all of $P \geq 0.207$, Table 3), and Morisita index (Paired $t$ test and all $P$ values $\geq 0.225$, Table 5). The calculated values of $b_{0}, b_{1}$, and $b$ at $20^{\circ} \mathrm{C}$ overlapped with these values at 25 and $30^{\circ} \mathrm{C}$ (Tables 6,7 ). The fitted distribution models to frequency data at $20^{\circ} \mathrm{C}$ were the same as that at 25 or $30^{\circ} \mathrm{C}$ (Table 4). Therefore, these small moisture gradients did not influence the characterized insect distribution patterns.

The fact of calculated values or analysis supporting each other explained that adults were not randomly distributed. They had a highly clump distribution at low and medium densities and this aggregation behavior decreased with the increase of insect density. These conclusions were also consistent with the fact that insect numbers at one location did not correlate to that at other locations, adults prefer the top $0.7 \mathrm{~m}$ of the grain mass, and there was temporal continuity. Therefore, to estimate the density of adult 
T. castaneum in stored grain silos, the top $0.7 \mathrm{~m}$ of the grain bulk and the places with $T$. castaneum infested grain found in the previous sampling should be sampled.

Acknowledgments The authors thank the Natural Sciences and Engineering Research Council of Canada and the Canada Research Chairs Program for partial funding of this study.

\section{References}

1. Agresti A (2007) An introduction to categorical data analysis, 2nd edn. Wiley, Hoboken

2. Agresti A (2010) Analysis of ordinal categorical data, 2nd edn. Wiley, Hoboken

3. Amos TG, Waterhouse FL (1969) The distribution and biology of Tribolium castaneum (Coleoptera, Tenebrionidae) on temperature gradients varying in steepness. Entomol Exp Appl 12:44-52

4. Arnaud L, Lognay G, Verscheure M, Leenaers L, Gaspar C, Haubruge E (2002) Is dimethyldecanal a common aggregation pheromone of Tribolium flour beetles? J Chem Ecol 28:523-532

5. ASABE (2008) ASABE standards 2008: standards, engineering practices, data. American Society of Agricultural and Biological Engineers, St. Joseph

6. Athanassiou CG, Kavallieratos NG, Palyvos NE, Buchelos CT (2003) Three-dimensional distribution and sampling indices of insects and mites in horizontally-stored wheat. Appl Entomol Zool 38:413-426

7. Boak CRB, Wade MJ (1984) Population of the red flour beetle Tribolium castaneum (Coleoptera: Tenebrionidae) differ in their sensitivity to aggregation pheromones. Environ Entomol 13: 1182-1185

8. Campbell RC (1989) Statistics for biologists, 3rd edn. Cambridge University Press, Cambridge

9. Davis PM (1994) Statistics for describing populations. In: Pegigo LP, Buntin GD (eds) Handbook of sampling methods for arthropods in agriculture. CRC Press, Boca Raton, pp 33-54

10. Ellsbury MM, Woodson WD, Clay SA, Malo D, Schumacher J, Clay DE, Carlson CG (1998) Geostatistical characterization of the spatial distribution of adult corn rootworm (Coleoptera: Chrysomelidae) emergence. Environ Entomol 27:910-917

11. Faustini DL, Burkholder WE, Laub RJ (1981) Sexually dimorphic setiferous sex patch in the male red flour beetle, Tribolium castaneum (Herbst) (Coleoptera: Tenebrionidae): site of aggregation pheromone production. J Chem Ecol 7:465-480

12. Goovaerts P (1997) Geostatistics for natural resources evaluation. Oxford University Press, New York

13. Graham WM (1958) Temperature preference determinations using Tribolium. Anim Behav 6:231-237

14. Green RH (1970) On fixed precision level sequential sampling. Res Popul Ecol 12:249-251

15. Hagstrum DW (2000) Using five sampling methods to measure insect distribution and abundance in bins storing wheat. J Stored Prod Res 36:253-262

16. Hagstrum DW, Flinn PW, Caffney JJ (1998) Temperature gradient on Tribolium castaneum (Coleoptera: Tenebrionidae) adult dispersal in stored wheat. Environ Entomol 27:123-129

17. Iwao S (1968) A new regression method for analyzing the aggregation pattern of animal populations. Res Popul Ecol 10:1-20

18. Iwao $\mathrm{S}$ (1977) The $\dot{m}-m$ statistics as a comprehensive method for analyzing spatial pattern of biological populations and its application to sampling problems. In: Morisita M (ed) Studies on estimating population density, biomass, and productivity in terrestrial animals. University of Tokyo Press, Tokyo, pp 21-46

19. Jian F, Jayas DS, White NDG (2005) Movement of Tribolium castaneum (Coleoptera: Tenebrionidae) adults in response to temperature gradients in vertical and horizontal wheat and corn columns. J Econ Entomol 98:1413-1419

20. Jian F, Larson R, Jayas DS, White NDG (2011) Three dimensional spatial distribution of adults of Cryptolestes ferrugineus (Coleoptea: Laemophloeidae) in stored wheat under different temperatures, moisture contents, and adult densities. J Stored Prod Res 47:293-305

21. Keeling MJ (2000) Simple stochastic models and their power law type behavior. Theor Popul Biol 58:21-31

22. Kitron U, Kazmierczak JJ (1997) Spatial analysis of the distribution of Lyme disease in Wisconsin. Am $\mathrm{J}$ Epidemiol 145:558-566

23. Langer GS, Young AM (1976) Habitat selection by flour beetles in complex environment. Physiol Zool 46:297-309

24. Lloyd M (1967) Mean crowding. J Anim Ecol 36:1-30

25. Lothrop HD, Reisen WK (1999) A geographical information system to manage mosquito and arbovirus surveillance and control data in the Coachella Valley of California. J Am Mosq Control Assoc 15:299-307

26. Mills R, Pederson J (1990) A flour mill station manual. Eagan Press, St. Paul

27. Nansen C, Liebhold A, Tobin P (2006) Counter mapping and number of point observations. J Econ Entomol 99:599-600

28. Ogden JG (1969) Aspects of dispersal in Tribolium flour beetles. Physiol Zool 43:124-131

29. Rossi RE, Mulla DJ, Journel AG, Franz EH (1992) Geostatistical tools for modeling and interpreting ecological spatial dependence. Ecol Monogr 62:277-314

30. Ryan PA, Lyons SA, Alsemgeest D, Thomas P, Kay BH (2004) Spatial statistical analysis of adult mosquito (Diptera: Culicidae) counts: an example using light trap data, in Redland shire, southeastern Queensland, Australia. J Med Entomol 41: $1143-1156$

31. SAS Institute (2008) SAS user guider, release 9.2. Cary

32. Senthilkumar T, Jayas DS, White NDG, Freund MS, Shafai C, Thomson DJ (2012) Characterization of volatile organic compounds released by granivorous insects in stored wheat. J Stored Prod Res 48:91-96

33. Sinha RN, Waters FL (1985) Insect pests of flour mills, grain elevators and feed mills and their control. Agriculture Canada Publication No. 1776. Canadian Government Publishing Center, Ottawa

34. Southwood TRF (1978) Ecological methods with particular reference to the study of insect populations, 2nd edn. Wiley, New York

35. Subramanyam B, Hagstrum DW, Meagher RL, Burkness EC, Hutchison WD, Naranjo SE (1997) Development and evaluation of sequential sampling plans for Cryptolestes ferrugineus (Stephens) (Coleoptera: Cucujidae) infesting farm-stored wheat. J Stored Prod Res 33:321-329

36. Surtees G (1963) Laboratory studies on dispersion behaviour of adults beetle in grain. III. Tribolium castaneum (HBST.) (Coleoptera, Tenebrionidae) and Cryptolestes ferrugineus (Steph.) (Coleoptera, Cucujidae). Bull Entomol Res 54:297-306

37. Surtees G (1964) Laboratory studies on dispersion behaviour of adults beetle in grain. VI. Three-dimensional analysis of dispersion of five species in a uniform bulk. Bull Entomol Res $55: 161-171$

38. Surtees G (1964) Laboratory studies on dispersion behaviour of adults beetle in grain. VIII. Spontaneous activity in three species and a new approach to the analysis of kinesis mechanisms. Anim Behav 12:374-377 
39. Surtees G (1965) Biological significance and practical implications of behaviour patterns determining the spatial structure of insect populations in stored grain. Bull Entomol Res 56:201-213

40. Systat Software Inc. (2009) Sigmaplot 11.2 user's guide, part 2statistics. San Jose

41. Taylor LR (1984) Assessing and interpreting the spatial distribution of insect population. Annu Rev Entomol 29:321-358

42. Tobin PC (2004) Estimation of the spatial autocorrelation function: consequences of sampling dynamic populations in space and time. Ecography 27:767-775
43. White NDG, Loschiavo SR (1986) Effects of insect density, trap depth, and attractants on the capture of Tribolium castaneum (Coleoptera: Tenebrionidae) and Cryptolestes ferrugineus (Coleoptera: Cucujidae) in stored wheat. J Econ Entomol 79:1111-1117

44. Zimmerman RH, Garris GI (1987) Use of Taylor's power law in examining the spatial distribution of free-living Boophilus microplus (Acari: Ixodidae) larvae in pastures in Puerto Rico. Exp Appl Acarol 3:207-211 